\title{
Neonatal and Adult Neurogenesis Provide Two Distinct Populations of Newborn Neurons to the Mouse Olfactory Bulb
}

\author{
Morgane Lemasson, ${ }^{1}$ Armen Saghatelyan, ${ }^{1}$ Jean-Christophe Olivo-Marin, ${ }^{2}$ and Pierre-Marie Lledo ${ }^{1}$ \\ ${ }^{1}$ Laboratory of Perception and Memory, Centre National de la Recherche Scientifique, Unité de Recherche Associée 2182, and ${ }^{2}$ Quantitative Image Analysis \\ Unit, Centre National de la Recherche Scientifique, Unité de Recherche Associée 2582, Pasteur Institute, 75724 Paris Cedex 15, France
}

In mammals, the olfactory bulb $(\mathrm{OB})$ constitutes one of two regions of the postnatal brain with continuous neurogenesis throughout life. Despite intense explorations of neuronal replacement in the adult $\mathrm{OB}$, little is known about the mechanisms that operate at earlier postnatal stages. This question is particularly pertinent, because the majority of local interneurons are born in the neonate, when olfaction controls vital functions. Here, we analyzed the recruitment of newborn cells to the granule cell (GC) layer (GCL) and found that the postnatal mouse $\mathrm{OB}$ is supplied with two spatiotemporally distinct populations of newborn interneurons.

Early born [postnatal day 3 (P3) to P7] GCs constitute a threefold larger population compared with those generated later (P14-P60), and some of them are produced locally within the $\mathrm{OB}$ itself. Newborn interneurons generated at $\mathrm{P} 3-\mathrm{P} 7$ were predominantly targeted to the external edge of the GCL, whereas newly generated cells were positioned deeper in older mice. Additionally, although $\sim 50 \%$ of adult newborn cells were eliminated within a few weeks of reaching the $\mathrm{OB}$, almost the entire population of early born GCs survived until adulthood. Importantly, early olfactory experience specifically modifies the number of newborn GCs in neonates but leaves unaltered the amount of neurons generated during adulthood. Together, these results demonstrate that early postnatal neurogenesis endows the neonate bulbar circuit with newborn GCs that differ morphologically and functionally from those produced in the adult.

Key words: BrdU; cell death; interneurons; migration; postnatal development; proliferation

\section{Introduction}

In most structures of the mammalian CNS, neurons are produced during a discrete time window, which ceases shortly after birth. In agreement with this scenario, the projection neurons of the olfactory bulb $(\mathrm{OB})$, the mitral and tufted cells, are generated during embryonic life (Hinds, 1968; Bayer, 1983). In contrast, neurogenesis of the local circuit neurons of the OB [periglomerular and granule cells (GCs)] is not completed at the end of the embryonic period but continues throughout life (Altman and Das, 1966; Hinds, 1968; Altman, 1969; Kaplan and Hinds, 1977; Rosselli-Austin and Altman, 1979; Bayer 1983; Luskin, 1993; Lois and Alvarez-Buylla, 1994). Postnatal bulbar interneurons derive from neuronal stem cells located in the subventricular zone (SVZ) of the lateral ventricles (Doetsch et al., 2002) from where they migrate to the $\mathrm{OB}$ along the rostral migratory stream (RMS)

\footnotetext{
Received 0ct. 19, 2004; revised May 30, 2005; accepted June 4, 2005.

This work was supported by the Pasteur Institute, the Ministère Délégué à la Recherche et aux Nouvelles Technologies (Actions Concertées Incitatives Biologie du Développement et Physiologie Intégrative, 2003), and the Fondation pour la Recherche sur le Cerveau. A.S. was supported by a postdoctoral fellowship from Association Française contre les Myopathies, and M.L. was supported by a PhD fellowship from the Fondation pour la Recherche Médicale. We thank Matt Grubb and Kerren Murray for critical comments on this manuscript. We also thank the Plate-forme d'Imagerie Dynamique for technical assistance.

Correspondence should be addressed to Pierre-Marie Lledo, Laboratory of Perception and Memory, Centre $\mathrm{Na}$ tional de la Recherche Scientifique, Unité de Recherche Associée 2182, Pasteur Institute, 25 rue du Docteur Roux, 75 724 Paris Cedex 15, France. E-mail: pmlledo@pasteur.fr.

D0I:10.1523/JNEUROSCI.1114-05.2005

Copyright $\odot 2005$ Society for Neuroscience $\quad$ 0270-6474/05/256816-10\$15.00/0
}

(Luskin, 1993; Lois and Alvarez-Buylla, 1994). Recently, it has been demonstrated that small numbers of newborn cells also originate in the RMS (Gritti et al., 2002; Pencea and Luskin, 2003). In the bulb, the newborn cells ascend radially into the overlying granule and glomerular cell layers to become, respectively, GCs and periglomerular interneurons (Altman, 1969; Betarbet et al., 1996; Belluzzi et al., 2003; Carleton et al., 2003). Despite the recent progress in characterizing the mode of migration of neuroblasts and their integration into the $\mathrm{OB}$ circuit, their functional contribution to the olfactory system remains essentially unknown. It is currently assumed that GCs produced postnatally constitute a uniform population of bulbar interneurons that is subjected to neuronal replacement throughout life. Yet neurons recruited at different postnatal stages could provide the $\mathrm{OB}$ with distinct classes of bulbar interneurons that might exert different functions depending on their time of generation.

The fact that new neurons can be continually generated in the postnatal brain raises a number of key questions about their morphofunctional properties. Do the newborn neurons produced at different postnatal ages exhibit distinct properties according to their sites of production along the SVZ-OB pathway? Are newly generated interneurons targeted to distinct bulbar regions according to their birthdates? Do newborn neurons produced at early and late postnatal stages degenerate within a few weeks of formation, or do they survive longer? Finally, do newborn neurons produced at different ages play distinct functional roles in 
the olfactory bulb neuronal network? The implications of these questions are manifold: it concerns the mechanisms that may be involved in the initial formation of $\mathrm{OB}$ circuits, and, in contrast, their plasticity and capacity for reorganization in the adult. To address these questions, we analyzed the precise contribution of postnatal neurogenesis to the developing and adult OB. Because the large majority of SVZ/RMS progenitors give rise to newborn neurons located in the granule cell layer (GCL), we concentrated our investigation specifically to this population. The patterns of cell proliferation, integration, fate determination, and survival of newborn neurons as well as the functional contributions of newly generated cells to the OB network were closely examined in mice from postnatal day 3 (P3) to P60. Our data reveal that early neurogenesis provides the developing $\mathrm{OB}$ with a population of newborn GCs that differs substantially from that produced during adulthood. Early and late postnatal neurogenesis therefore fulfill distinct functions in the OB network.

\section{Materials and Methods}

Subjects. Male mice used for this study were derived from C57BL/6 breeding pairs. Pregnant females were housed individually in polypropylene cages $(34 \times 29 \times 17 \mathrm{~cm})$ lined with wood chips and were kept in a temperature-controlled $\left(20 \pm 1^{\circ} \mathrm{C}\right)$ and light-controlled (8:00 A.M. to 8:00 P.M.) room. Food and water were available ad libitum. The day of birth was considered P0. In some experiments, pups were reared in a citral-scented environment from embryonic day 18 (E18) to P9 under a vented hood. To prevent any odor-induced change in the mothers' behavior, pregnant females were placed in a citral-odorized condition $3 \mathrm{~d}$ before expected parturition (E18). The olfactory environment was altered daily by adding citral (100 $\mu$; Sigma-Aldrich, St. Louis, MO) directly to the animals' shavings. Control litters were housed under similar conditions, but only saline solution was placed in their bedding. At P9, pups and their mother were kept free of citral odor in a clean home cage. All experimental procedures were performed in accordance with the Society for Neuroscience and European Union guidelines and were approved by our institutional animal care and utilization committees.

5-Bromo-2'-deoxyuridine injections. To determine the quantity of newly generated cells, the DNA synthesis marker 5-bromo-2'deoxyuridine (BrdU; Sigma) was administered intraperitoneally (50 $\mathrm{mg} / \mathrm{kg}$ body weight) and dissolved in a sterile solution of $0.9 \% \mathrm{NaCl}$ and $1.75 \% \mathrm{NaOH}(0.4 \mathrm{~N})$. A single dose of BrdU was injected into mice at P3, P7, P14, P21, and P60. BrdU-containing cells were detected by immunohistochemistry after different survival times. Morphological analyses after short survival times $(2 \mathrm{~h})$ revealed the quantity of cellular proliferation in the SVZ-OB pathway, whereas longer survival times (3-4 weeks) revealed migration, distribution, and survival processes of newborn cells.

To study the effect of early olfactory experience on postnatal neurogenesis, a single dose of $\mathrm{BrdU}$ was injected into mice for four consecutive days from P4 to P7, and mice were killed at P27 ( $20 \mathrm{~d}$ after receiving the final BrdU injection). Four BrdU injections repeated every $2 \mathrm{~h}$ were administered to adult animals (P45), and BrdU-containing cells were detected by immunohistochemistry after the same survival time as in younger mice. To prevent possible differences between litters, an equivalent number of pups from the same litter was exposed to the different conditions.

Immunohistochemistry. Mice were given an overdose of sodium pentobarbital (100 mg/kg body weight; Sanofi, Libourne, France) and perfused transcardially with saline solution $(0.9 \% \mathrm{NaCl})$ containing heparin $\left(5 \times 10^{3} \mathrm{U} / \mathrm{ml}\right)$ at $37^{\circ} \mathrm{C}$, followed by $4 \%$ paraformaldehyde prepared in $0.1 \mathrm{~m}$ phosphate buffer, $\mathrm{pH}$ 7.3. The brain was dissected out and immersed for $48 \mathrm{~h}$ in the same fixative at $4^{\circ} \mathrm{C}$. Immunohistochemistry was performed on $40-\mu \mathrm{m}$-thick free-floating serial coronal and parasagittal sections cut with a vibrating microtome (VT1000S; Leica, Nussloch, Germany) and collected in PBS $(0.1 \mathrm{M})$. Sections were first pretreated with $0.2 \%$ Triton $\mathrm{X}-100$ for $2 \mathrm{~h}$, and DNA was denatured with $2 \mathrm{~N} \mathrm{HCl}$ for 30 $\min$ at $37^{\circ} \mathrm{C}$. The primary antibodies used were rat monoclonal antiBrdU antibody (1:200; Harlan Sera-Lab, Loughborough, UK) and mouse monoclonal anti- neuronal-specific nuclear protein (NeuN) antibody (1:200; Chemicon, Temecula, CA). Incubation with these primary antibodies at $4^{\circ} \mathrm{C}$ was followed by a $2 \mathrm{~h}$ incubation at room temperature with corresponding biotinylated secondary antibodies (1:200; Vector Laboratories, Burlingame, CA) and by a $1 \mathrm{~h}$ incubation with avidin-biotin complex (ABC kit; Vector Laboratories). The reaction was revealed by diaminobenzidine (DAB; 0.05\%; Vector Laboratories). Double-labeled immunofluorescence was performed with the following fluorescent secondary antibodies: Alexa 568-conjugated goat anti-rat IgG antibody and Alexa 488-conjugated goat anti-mouse IgG antibody (both at 1:500). Sections were analyzed using either a standard microscope (BX51; Olympus, Tokyo, Japan) for the peroxidase staining or a confocal microscope (Zeiss, Thornwood, NY) for double labeling.

To detect DNA fragmentation, terminal deoxynucleotidyl transferasemediated biotinylated UTP nick-end labeling (TUNEL) was performed in $40-\mu \mathrm{m}$-thick free-floating serial coronal sections of the olfactory bulb of 1- and 3-month-old animals. The slices were incubated for $1 \mathrm{~min}$ in equilibrium buffer (Serological Corporation, Purchase, NY) followed by $1 \mathrm{~h}$ at $37^{\circ} \mathrm{C}$ in a solution containing terminal deoxynucleotidyl transferase and digoxigenin nucleotides (Serological Corporation). After stopping the reaction with stop solution (Serological Corporation), sections were washed and incubated for $1 \mathrm{~h}$ with a peroxidase-labeled antidigoxigenin secondary antibody (Serological Corporation) at room temperature. The staining was revealed by $\mathrm{DAB}$ and visualized using a standard microscope (BX51; Olympus).

Quantification. On every third $40 \mu \mathrm{m}$ section, BrdU-immunostained nuclei were visualized through a $10 \times$ objective lens and automatically numbered by a computer program that used an undecimated wavelet decomposition of the image for detecting objects. The program recognizes nuclei by correlating and thresholding wavelet coefficients at different scales of analysis (Olivo-Marin, 2002). Following the interactive drawing of the internal (border of the RMS) and external [border of the mitral cell body layer (MCL)] contours of the GCL and of its major axis, the program computes, for each nucleus, the distances $d_{\text {in }}$ and $d_{\text {out }}$ to the borders and the angle $\theta$ of the radius with the major axis. This axis goes through the "center of mass" of the RMS and the most ventral point of the GCL. The external border of the RMS was easily distinguishable with high magnifications, because cytoarchitectures of these layers were quite distinct. Measurements are given as the normalized distance, $D_{\text {norm }}=$ $d_{\text {out }} /\left(d_{\text {in }}+d_{\text {out }}\right)$, of each nucleus to the external border and as the clockwise value of the angle. The values obtained for $D_{\text {norm }}$ and $\theta$ were grouped into $10 \mathrm{rim}$ categories corresponding to normalized distance steps of 0.1 and into 24 angular quadrants of $15^{\circ}$ (see Fig. $4 a, e$ ). The number of BrdU-labeled cells in each part of the GCL was then represented as both a percentage and a density.

$\mathrm{TUNEL}^{+}$cells in the GCL were counted with a $20 \times$ objective lens. The percentages of BrdU/NeuN double-immunostained cells were obtained by analyzing three-dimension (3D)-reconstructed $\mathrm{BrdU}^{+}$nuclei in the $x-z$ and $y-z$ orthogonal projections for the presence of NeuN.

Behavioral analysis. The olfactory test consisted of a two-odor choice task both for young and adult mice, but different test apparatuses were used depending on the age of the animals. For young mice, the test was conducted in a transparent plastic chamber $(36 \times 24 \times 16 \mathrm{~cm})$ divided into two olfactory stimulus environments by a solid separator $(3.5 \mathrm{~cm})$. One of the preference chambers contained clean shavings, and the other one an equal volume of citral-scented or (-)carvone-scented (Fluka, Buchs, Switzerland) shavings ( $24 \mathrm{~g}$ of shavings with $5 \mu \mathrm{l}$ of odor). The shavings arena was covered by a stainless-steel grate with a plastic mesh, which allowed olfactory but not gustatory or tactile cues to pass from the shavings, and in which the pups roamed freely during the test session. The preference chamber had a removable transparent cover open on the top $(27 \times 15 \mathrm{~cm})$ to minimize stagnancy of odor. For adult animals, a larger apparatus was used. The testing apparatus consisted of a plastic chamber $(73 \times 11 \times 22 \mathrm{~cm})$ divided in half by a neutral zone. The olfactory stimulus was placed at each end of this arena. As for young animals, adults had to choose between clean shavings versus citral- or (-)carvone-scented shavings ( $24 \mathrm{~g}$ of shavings with $5 \mu \mathrm{l}$ of odor).

Each animal was tested in preference tests at P8 and P60. At the beginning of the session, the mouse was introduced in the center of the grate on 
the neutral zone, midway between the two olfactory stimuli. The cover was replaced, and the mouse started exploring both preference chambers. We recorded the time that the animals spent in half of the apparatuses during a session test lasting $6 \mathrm{~min}$. Odor investigation was scored only if the anterior snout of the animals was reaching one side. The orientation of the animal was changed between trials to control for any turning bias. The test chamber was cleaned and dried before submitting each new mouse to testing conditions.

Statistical analysis. All experiments were performed blind to the experimental conditions. Statistical differences were assessed either by nonparametric Mann-Whitney $U$ tests or by one-way Kruskal-Wallis tests for distribution analysis. Differences in olfactory preference were assessed using an independent Student's $t$ test. The level of significance was set at $p<0.05$.

\section{Results}

\section{Local cell proliferation in the $\mathrm{OB}$ of postnatal mice}

Although the adult interneurons produced principally in the SVZ (Doetsch et al., 2002) and partly in the RMS (Gritti et al., 2002; Pencea and Luskin, 2003) populate an already mature OB circuit, those from the neonate integrate into a developing OB. Thus, the quantity and the distribution of postnatally generated bulbar interneurons, as well as their site of generation along the SVZ-OB pathway, might differ substantially during development. To investigate whether newborn cells might be produced locally within the developing OB itself, we quantified the number of proliferating cells in the GCL across different ages. To exclusively label locally born cells, we injected a single dose of BrdU in 3-, 7-, 14-, 21-, and 60-d-old mice and processed the brains for immunochemistry $2 \mathrm{~h}$ after the injection ("short-survival group"). Considerable differences in the density of $\mathrm{BrdU}^{+}$cells in the GCL were observed across ages. As seen in Figure $1 a$, the density of BrdU-labeled nuclei was high at P3 and declined thereafter (Fig. $1 b-e$ ) until P60 (Kruskal-Wallis test; $H_{(4,16)}=14.4 ; p=0.006$ ). More precisely, the number of newborn cells observed in the GCL sharply declined between P3 and P7 (a reduction of 75\%) and between P7 and P14 (a reduction of 90\%), reaching significance with a multiple comparison test for $\mathrm{P} 3$ versus $\mathrm{P} 21$ and $\mathrm{P} 3$ versus P60 groups ( $p=0.04$ and $p=0.02$, respectively) (Fig. $1 f$ ). Thus, results from the short-survival group identified an early wave of local proliferation within the $\mathrm{OB}$ that becomes negligible soon after birth.

\section{Age-dependent recruitment of newborn cells in the GCL}

To investigate the time required for newborn cells to reach the GCL from the SVZ across different ages, we administered a single pulse of BrdU to mice aged P3-P60 and analyzed their brains at various time points after the injection. Most neonatal, juvenile, and adult newborn cells derive from a germinal layer outside the bulb and migrate a long distance to reach their final destinations (Fig. 2a). The critical period needed for newborn cells to complete their tangential and radial migrations was defined in sagittal (Fig. 2a) and coronal (Fig. 2b) sections by determining the postBrdU injection time by which virtually no $\mathrm{BrdU}^{+}$cells could be seen in the SVZ-RMS migratory pathway. The vast majority of $\mathrm{BrdU}^{+}$cells generated at P3 reach the GCL $26 \mathrm{~d}$ after BrdU injection, and almost no newborn cells were found in the core of the $\mathrm{OB}\left(\mathrm{RMS}_{\mathrm{OB}}\right) 28 \mathrm{~d}$ after injection (Fig. 2b,c). Surprisingly, the time required for the $\mathrm{RMS}_{\mathrm{OB}}$ to empty of $\mathrm{BrdU}^{+}$cells diminished progressively with age. Newborn cells generated in P3, P7, P14, P21, and P60 mice reached the GCL after 28, 26, 24, 22, and $20 \mathrm{~d}$ after the BrdU injection, respectively (Fig. $2 b$ ). Thus, despite the growing distance between the production zone and the integration site in the developing forebrain, newborn cells reach their target faster in young adults compared with neonates.
P3

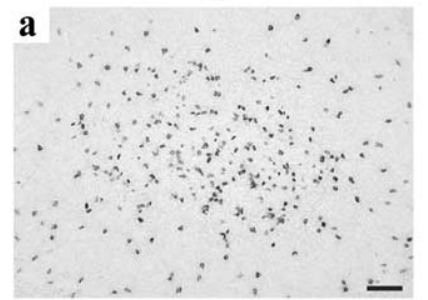

P14

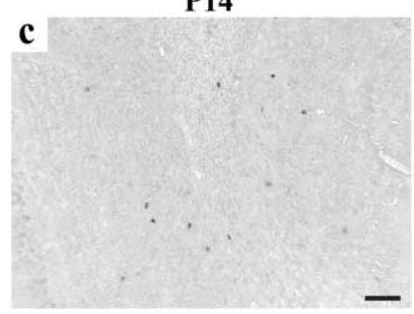

P60

e
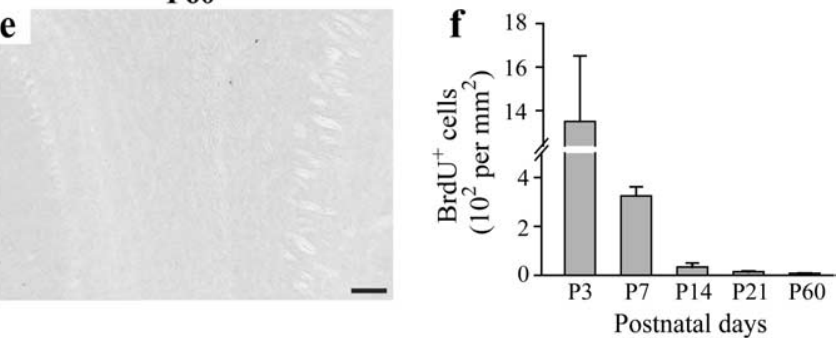

Figure 1. Local cellular proliferation in the postnatal olfactory bulb decreases with age. $\boldsymbol{a}-\boldsymbol{e}$, Representative images of $\mathrm{BrdU}^{+}$cells located in the granule cell layer of 3-d-old $(\boldsymbol{a})$, 7-d-old (b), 14-d-old (c), 21-d-old (d), and 60-d-old (e) mice. $\boldsymbol{f}$, Mean density of BrdU ${ }^{+}$cells (per $\mathrm{mm}^{2}$ ) in the granule cell layer determined $2 \mathrm{~h}$ after a single injection of $\mathrm{BrdU}(n=2-4$ animals per group). Scale bar, $50 \mu \mathrm{m}$.

With the above results, we evaluated the quantity and the distribution of newborn cells generated in P3, P7, P14, P21, and P60 mice. Animals were killed 28, 26, 24, 22, and 20 d, respectively, after a single BrdU injection ("long-term survival group"). In agreement with previous results (Hinds, 1968), the average number of $\mathrm{BrdU}^{+}$profiles changed across ages (Kruskal-Wallis test; $H_{(4,22)}=17.2 ; p=0.001$ ) (Fig. $3 a$ ) and reached a peak at P7 $\left(1275 \pm 116\right.$ cells $/ \mathrm{mm}^{2}$ ) before declining to $304 \pm 3$ cells $/ \mathrm{mm}^{2}$ at P60 (Fig. 3a). When the distribution patterns of $\mathrm{BrdU}^{+}$cells were analyzed throughout the rostrocaudal axis of the $\mathrm{OB}$, we found that newborn cells were evenly distributed across ages throughout the length of the bulb (Fig. $3 b$ ).

The population of newborn cells located in the GCL and quantified in the long-survival group might include cells generated locally in the OB and those issued from the SVZ/RMS system. Therefore, to quantify more precisely the contribution of each population, a neurogenic index was established that takes local production into account by determining the ratio of the number of $\mathrm{BrdU}^{+}$cells in the $\mathrm{OB}$ of the long-term survival group to that of the short-term group (Fig. 3). We observed a gradual increase in the number of SVZ/RMS-generated cells during development (Fig. 3c), as a result of the negligible local proliferation in older animals (Fig. 1). Thus, newly generated cells are evenly distributed across the anteroposterior axis of the $\mathrm{OB}$, and the relative contribution of SVZ versus bulbar neurogenesis increases with age. 


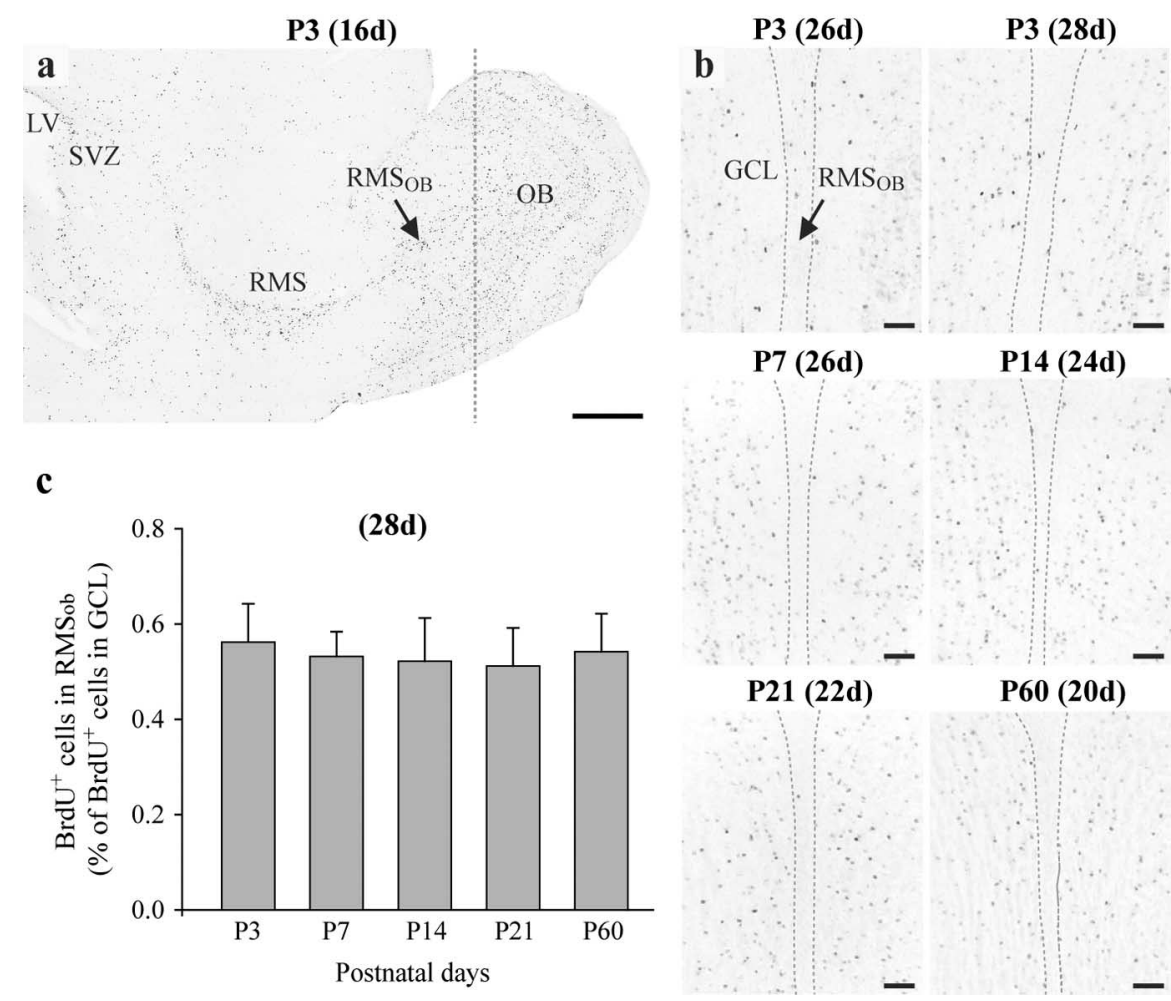

Figure 2. Age-dependent changes in the migration duration required for neuroblasts to reach the OB. $\boldsymbol{a}$, Representative images of a sagittal section of a 19-d-old mouse brain demonstrating the pattern of distribution of BrdU ${ }^{+}$cells generated at $P 3$ along the SVZ/OB pathway. LV, Lateral ventricle. $\boldsymbol{b}$, Coronal sections of the OB of animals injected with BrdU at P3, P7, P14, P21, and $\mathrm{P} 60$ and killed following different survival periods as indicated in the parentheses. The coronal sections were taken at the leve of the dotted line indicated in the sagittal section in $\boldsymbol{a}$. Note the disappearance of BrdU ${ }^{+}$cells in the $\mathrm{RMS}_{\mathrm{OB}}$ following the chosen survival times. $\boldsymbol{c}$, Ratio of BrdU ${ }^{+}$cells in the $\mathrm{RMS}_{O B}$ to those in the OB. Scale bars: $\boldsymbol{a}, 500 \mu \mathrm{m} ; \boldsymbol{b}, 50 \mu \mathrm{m}$.
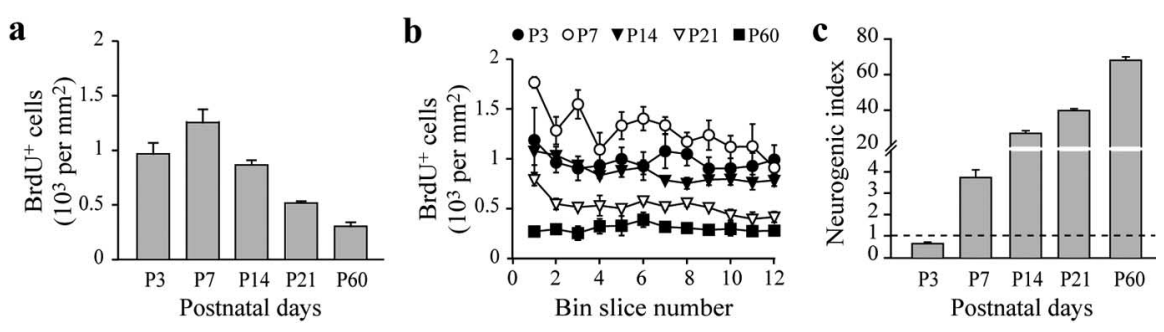

Figure 3. Age-dependent decreases in the density of surviving newly generated cells in the granule cell layer. $\boldsymbol{a}$, Mean number of BrdU ${ }^{+}$cells (per $\mathrm{mm}^{2}$ ) in the granule cell layer at different ages. Animals ( $n=3-5$ per group) were killed following a time window required to empty the RMS (as indicated in Fig. 2). $\boldsymbol{b}$, Mean number of BrdU ${ }^{+}$cells (per $\mathrm{mm}^{2}$ ) throughout the rostrocaudal axis of the OB. c, Ratio of SVZ/RMS-generated cells in the $O B$ after migration versus that of locally proliferating cells at different ages. Note that, because of the decreased local $O B$ proliferation with age, this ratio increases during postnatal development.

\section{Different spatial distribution of newborn cells in the GCL across ages}

Despite the fact that neonatal and adult neurogenesis provide a uniform distribution of newborn cells throughout the rostrocaudal axis of the OB, cells could still be preferentially targeted to superficial or deep parts of the GCL according to age. Thus, we explored the distribution of newborn cells in the long-term survival group using a precise stereological counting technique (Fig. 4a) (see Materials and Methods). When this distribution was calculated as a percentage of the entire population of $\mathrm{BrdU}^{+}$cells located in the GCL, pronounced differences in the distribution profiles were observed across ages (Kruskal-Wallis test for all of the bins; $H_{(4,22)}$, from 14.3 to 18.6 , depending on the bin; $p=$
0.001) (Fig. 4b). Cells produced at P3-P7 were preferentially targeted to the external portion of the GCL, including the MCL and the internal plexiform layer (IPL) (Fig. $4 b$, circles). Those generated between 14 and $21 \mathrm{~d}$ reached a maximum density just underneath the IPL (Fig. $4 b$, triangles). In contrast, adult-born cells were uniformly distributed from the MCL to the $\mathrm{RMS}_{\mathrm{OB}}$. Although these results demonstrate important age-dependent changes in the distribution profiles of newborn cells, they do not take into account differences in sampling areas within the same animal and across ages. To address this issue, we also evaluated the spatiotemporal distribution of the density of $\mathrm{BrdU}^{+}$cells per $\mathrm{mm}^{2}$ (Fig. $4 c$ ). In agreement with the above results based on absolute cell counts, this measure showed that newborn cells produced at P3-P7 were more densely located at the external edge of the GCL, whereas the distribution of newly generated neurons was densest in deeper portions of the GCL in older animals (Kruskal-Wallis test; $H_{(4,22)}$, from 16.3 to 18.6 , depending on bin; $p=0.002$ ) (Fig. $4 c$ ).

Figure $4 d$ shows clearly that local proliferation within the $\mathrm{OB}$ (closed circles) could not support this uneven distribution, except for the cells produced locally at P3. In 3-d-old pups, in which local neurogenesis is quite important, cells generated in the OB ( $2 \mathrm{~h}$ after BrdU exposure) tend to be systematically positioned in deep regions of the GCL, where the density of surviving newborn cells (28 d after BrdU exposure) was the lowest. Because it is not clear whether these locally produced cells can migrate to the superficial layer of the GCL over time, we excluded the P3 group from additional analysis. Although a similar tendency was observed in P7 pups, the level of local production (Fig. $4 d$, closed circles) was much lower compared with the overall population of $\mathrm{BrdU}^{+}$cells positioned in the GCL after long-term survival (Fig. $4 d$, open circles). This observation therefore cannot explain the distribution profile of surviving newborn cells at this age. Finally, from P14 to P60, local proliferation was clearly negligible compared with the amount of newborn cells positioned in the GCL after long-term survival time and, furthermore, it showed an even distribution. Local production of newborn neurons in the $\mathrm{OB}$ is therefore unlikely to contribute to the differences in cell distribution seen in the GCL with increasing age. Overall, we conclude that SVZ/RMS-derived cells produced between P3 and P7 are mainly targeted to the superficial regions of the GCL, whereas newly generated cells in older animals are predominantly found in deeper regions.

Because the recruitment of newborn cells to the GCL showed a clear internal-external gradient across ages, we further examined their spatiotemporal pattern of distribution in the ventral, 
medial, dorsal, and lateral parts of the GCL (here called ventrodorsal distribution). For this, we quantified the number of $\mathrm{BrdU}^{+}$ cells in a circular manner (Fig. 4e). Because of the shape of frontal sections, the areas containing the highest percentage of newborn cells were primarily located in the ventral and dorsal portions of the GCL (Fig. 4f). Nevertheless, when expressed as a cell density, the cellular distribution was uniform along both axes and across all different ages (Fig. $4 g$ ). Our results therefore clearly establish age-dependent differences in the distribution profiles of new cells in the internalexternal axis but not in the ventrodorsal or the rostrocaudal axes of the GCL.

\section{Apoptotic activity and cell fate in the} GCL of the neonate and adult $O B$

The gradients of neonatal and adult neurogenesis seen between the external and internal edge of the GCL could result from a specific targeting of newborn cells. Alternatively, they might originate from a regionalization of apoptotic activity. We thus determined whether the elimination of newly generated cells was homogeneous across ages. Using the TUNEL assay, which labels fragmented DNA with tagged nucleotides, we quantified programmed cell death in the GCL of 1- and 3-month-old mice. These ages correspond approximately to the time window for hypothesized cell elimination-induced changes in the distribution profiles of perinatal- and adult-derived newborn cells. In agreement with previous studies (Biebl et al., 2000; Najbauer et al., 2002; Petreanu and AlvarezBuylla, 2002; Winner et al., 2002; Mechawar et al., 2004), many TUNEL $^{+}$cells were found in the GCL in both the neonatal and the adult $\mathrm{OB}$ (Fig. 5a). Using stereological counting techniques, we found that both the density (Fig. 5b) and the distribution (Fig. 5c) of apoptotic cells were uniform and indistinguishable between the two groups, suggesting that differences in the internal-external distribution profiles of newborn cells might arise from differential targeting of newborn cells to the GCL rather than from their selective elimination. Additional experiments designed to measure the number of BrdU-labeled cells at different time points and specifically apoptotic signaling, such as those based on immunofluorescence of members of the caspase family, should clarify this issue.

To determine the proportion of newborn cells that mature into neurons in the developing $\mathrm{OB}$, we also evaluated the proportion of cells double labeled for BrdU and the neuronal marker NeuN. Analysis of $\mathrm{BrdU}^{+}$cells in the long survival group revealed that the majority of them ( $\sim 90 \%)$ also expressed the neuronal marker NeuN, indicating unambiguously their neuronal identity (Fig. $5 d$ ). The proportion of newborn neurons produced and integrated into the GCL remained constant from P7 to P60 (Fig. 5e).

\section{Different survival rates of newborn GCs in early and late postnatal neurogenesis}

It has been shown that cell death is a prominent factor regulating neurogenesis during earlier CNS development (Katz and Shatz, 1996; Voyvodic, 1996) as well as in the adult OB (Najbauer and b

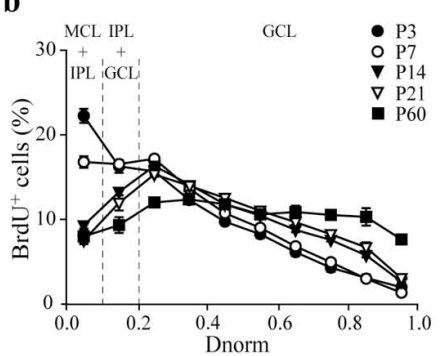

c
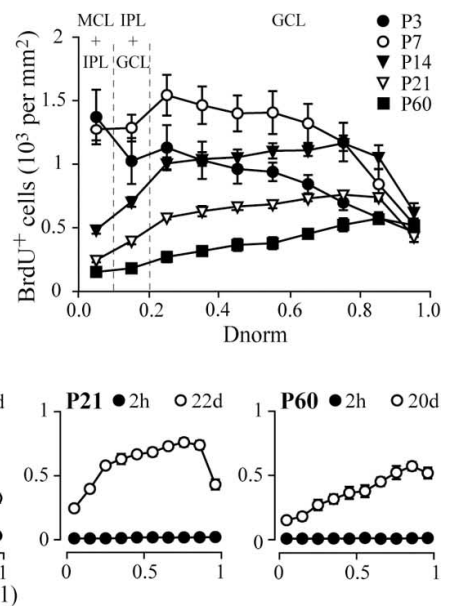

g

f
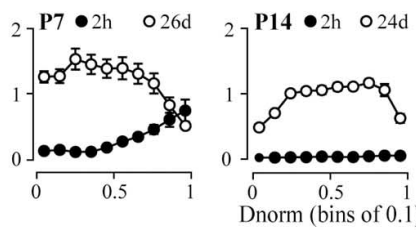
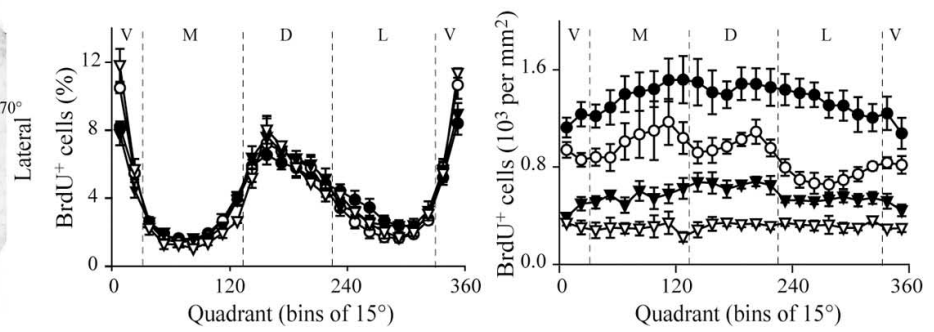
th

Figure 4. Targeting of newborn granule cells changes during bulbar development. $\boldsymbol{a}, \boldsymbol{e}$, Quantifying BrdU ${ }^{+}$cell distribution xternal distribution of BrdU ${ }^{+}$cells measured after short-term ( 2 h; black circles) or long-term (from 20 to $28 \mathrm{~d}$, depending on postnatal age of animals; white circles) survival. $\boldsymbol{f}, \boldsymbol{g}$, Distribution of $\mathrm{BrdU}^{+}$cells throughout the ventrodorsal axis expressed as percentage $(\boldsymbol{f})$ and density $(\boldsymbol{g})$. Note the equal distribution of BrdU ${ }^{+}$profiles across ages. Scale bars, $200 \mu \mathrm{m}$.

Leon, 1995; Fiske and Brunjes, 2001; Petreanu and Alvarez-Buylla, 2002; Winner et al., 2002; Mechawar et al., 2004). To evaluate the long-term persistence of newborn cells generated during an early postnatal period and in adulthood, we labeled cells with BrdU and quantified them at two different survival times: 22 and $60 \mathrm{~d}$ after BrdU injections (Fig. 6a). To increase the number of BrdU-labeled cells, four pulses of BrdU (one per day) were given to neonates (from P4 to P7) and young adult mice (P60). Remarkably, although half of the population of adult-generated GCs was eliminated during this time window (Fig. 6b) (Mann-Whitney $U$ test; $p=0.01$ ), the density of newborn GCs produced at early stages remained virtually unchanged (Fig. 6b) (Mann-Whitney $U$ test; $p>0.05$ ), revealing that the survival rate of newborn cells tightly depends on their birthdates.

As shown above, populations of newborn neurons produced at different ages are also targeted differently across the internalexternal axis of the GCL. To investigate whether this distinct distribution is preserved for the GC population showing longlasting survival, we evaluated their positioning in the GCL 22 and $60 \mathrm{~d}$ after BrdU injections. Remarkably, although the distributions of $\mathrm{BrdU}^{+}$cells produced in early $(\mathrm{P} 4-\mathrm{P} 7)$ and late $(\mathrm{P} 45)$ postnatal life were clearly distinct (Figs. $4 b, 6 c$ ), no differences were seen in the spatial distribution.

Early olfactory experience increases neurogenesis in neonates but not in young adults

So far, our data indicate significant differences in the GC populations produced at distinct postnatal stages regarding their site of 
a

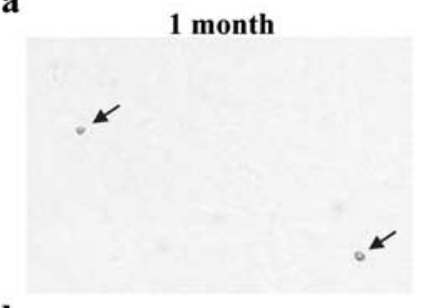

b

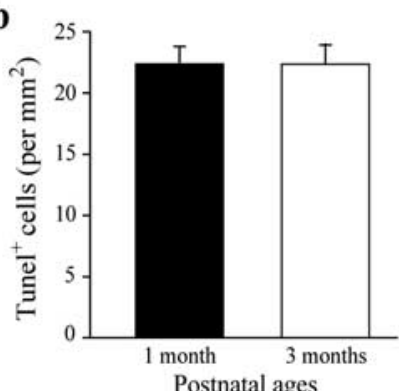

d

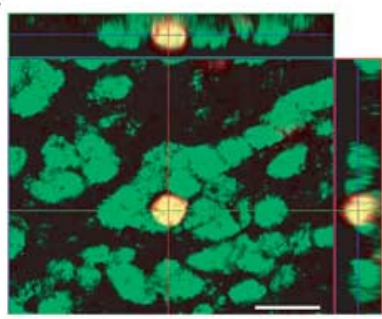

c
3 months
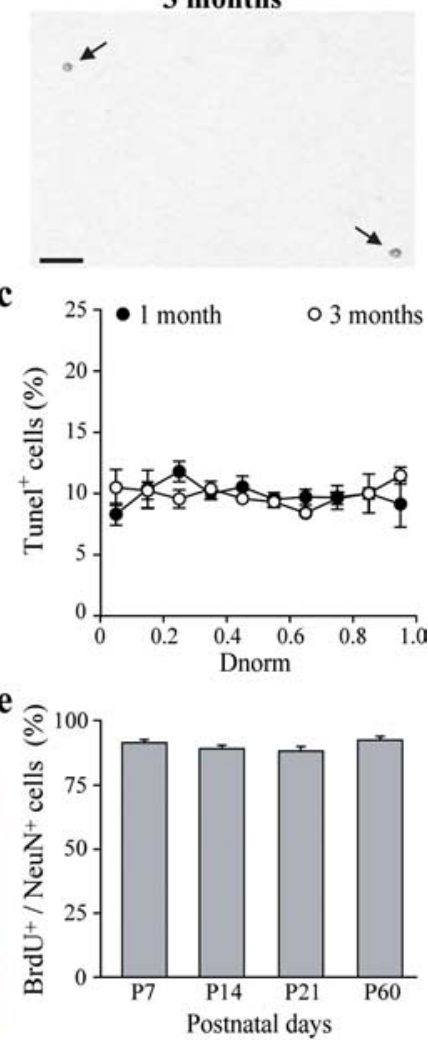

Figure 5. Cell death and fate of newborn cells remain constant in the olfactory bulb at different postnatal ages. $\boldsymbol{a}$, TUNEL ${ }^{+}$cells (arrows) in the middle of granule cell layer of 1-month-old (left) and 3-month-old (right) mice. $\boldsymbol{b}$, Number of apoptotic nuclei (per $\mathrm{mm}^{2}$ ) in the granule cell layer of young and adult mice. $c$, Distribution of TUNEL ${ }^{+}$cells (expressed as percentage) within the internal-external axis of the granule cell layer of 1 - and 3-month-old animals ( $n=4$ per group). $\boldsymbol{d}$, Example of a 3D-reconstructed BrdU ${ }^{+}$cell (red) colabeled with the neuronal marker NeuN (green) in the granule cell layer. Reconstructed orthogonal projections are presented as viewed in the $x-z$ (top) and $y-z$ (right) planes. e, Percentage of doublelabeled cells in the granule cell layer of 7-, 14-, 21-, and 60-d-old mice. More than 1000 cells, taken from three animals per group, were analyzed for each postnatal age. Scale bars: $\boldsymbol{a}, 20$ $\mu \mathrm{m} ; \boldsymbol{d}, 10 \mu \mathrm{m}$. Error bars indicate SEM.

production, their distribution, and their survival rate. Next, we investigated whether these differences could have some functional correlates. It is well established that presentation of an artificial odor during early postnatal life later induces marked preferences for this odor (Rodriguez Echandia et al., 1982; Shah et al., 2002). To investigate whether such early olfactory experience alters neurogenesis, pups were reared in the presence of citral or saline during $9 \mathrm{~d}$ between E18 and P9. Figure $7 a$ shows that control P8 pups avoided citral in a two-choice behavioral test, whereas those reared in a citral-rich environment (from birth to P9) showed a marked preference for citral (Fig. 7a, top). This effect was long-lasting, because citral-reared animals still expressed a significant preference for citral 2 months later. The specificity of this response was demonstrated by the absence of any preference when animals were tested with (-)carvon at P8 and P60 (Fig. 7a, top). Remarkably, rearing in a citral-rich environment not only induced a preference for this odor but also increased the number (Fig. $7 a$, bottom) and the density (data not shown) of newborn GCs produced at P4-P7. This increase was homogeneous in the $\mathrm{OB}$, because the rostrocaudal, internalexternal, and ventrodorsal distributions of newborn cells remained unchanged (Fig. $7 b$ ). Because early olfactory experience

$\mathbf{a}$

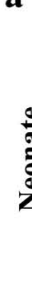

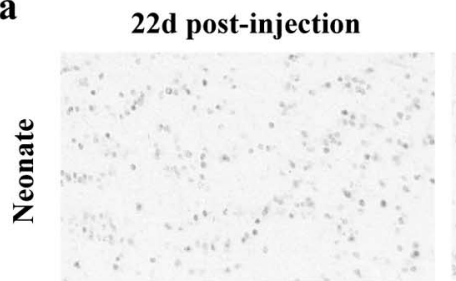

60d post-injection
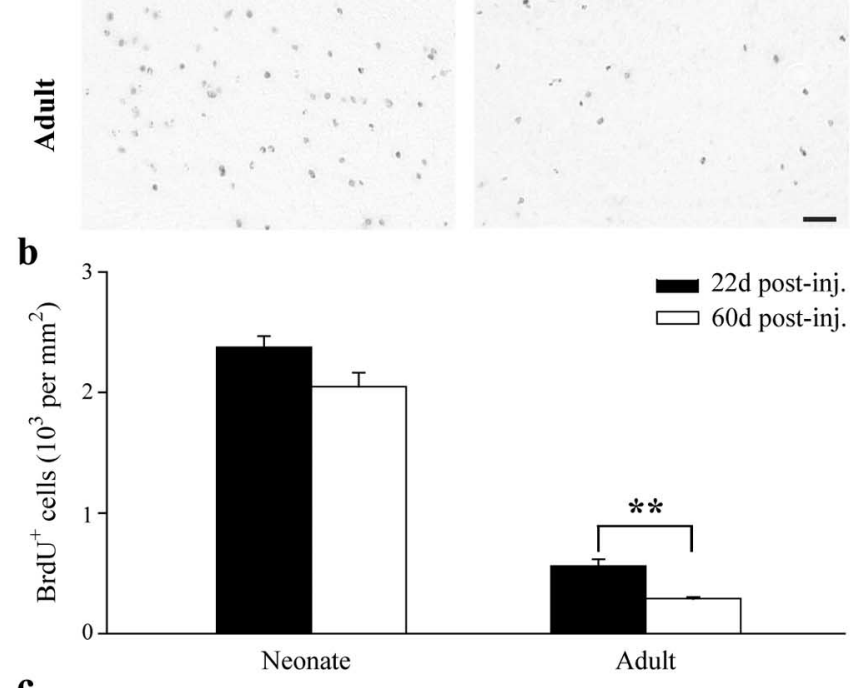

c
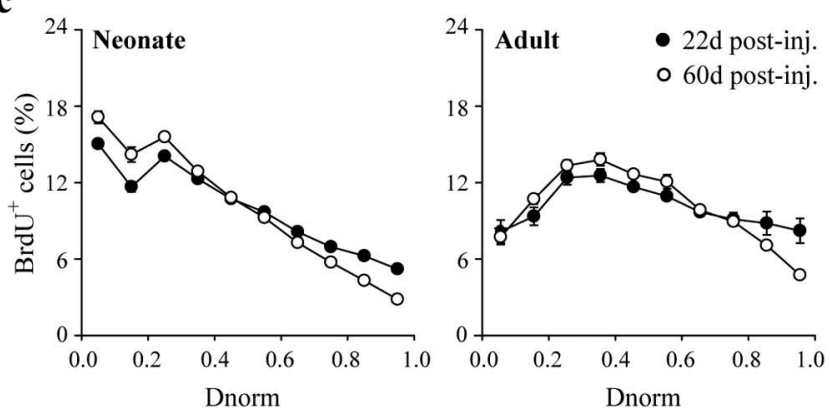

Figure 6. Different survival rates of newborn granule cells in the neonate and the adult olfactory bulb. $\boldsymbol{a}$, Photomicrographs of BrdU-immunostained sections of the granule cell layer in early postnatal (top; P4) and adult mice (bottom; P45) $22 \mathrm{~d}$ (left) and $60 \mathrm{~d}$ (right) after BrdU injections. $\boldsymbol{b}$, Corresponding densities of $\mathrm{BrdU}{ }^{+}$cells generated during different periods of life and allowed to survive for short ( $22 \mathrm{~d}$ ) and long (60 d) BrdU postinjection times ( $n=4-10$ animals per group). Note the differences in age-dependent persistence of newborn cells in young and adult mice. c, Distribution of early- and late-generated GCs following different survival periods. Note the distribution differences between early postnatal (P4) and adult animals (P45) versus the similar distributions observed following different survival periods. ${ }^{* *} p<$ 0.001. Scale bar, $20 \mu \mathrm{m}$. Error bars indicate SEM.

induced a long-lasting behavioral preference, neurogenesis was also evaluated in adult mice that had been exposed to a citral-rich environment as pups. We found that the number of GCs generated in adulthood was insensitive to early experience (Fig. 7a, bottom). Together, these results indicate that the formation of a long-lasting olfactory memory established during perinatal life is associated with an increase in the population of early but not late-born cells. Error bars indicate SEM.

\section{Discussion}

The present study describes differences in the properties of neonate- and adult-generated newborn GCs with respect to their origin, time required to reach the bulb, ultimate position attained in the GCL, and their survival rate. Our results also show that it is 
a

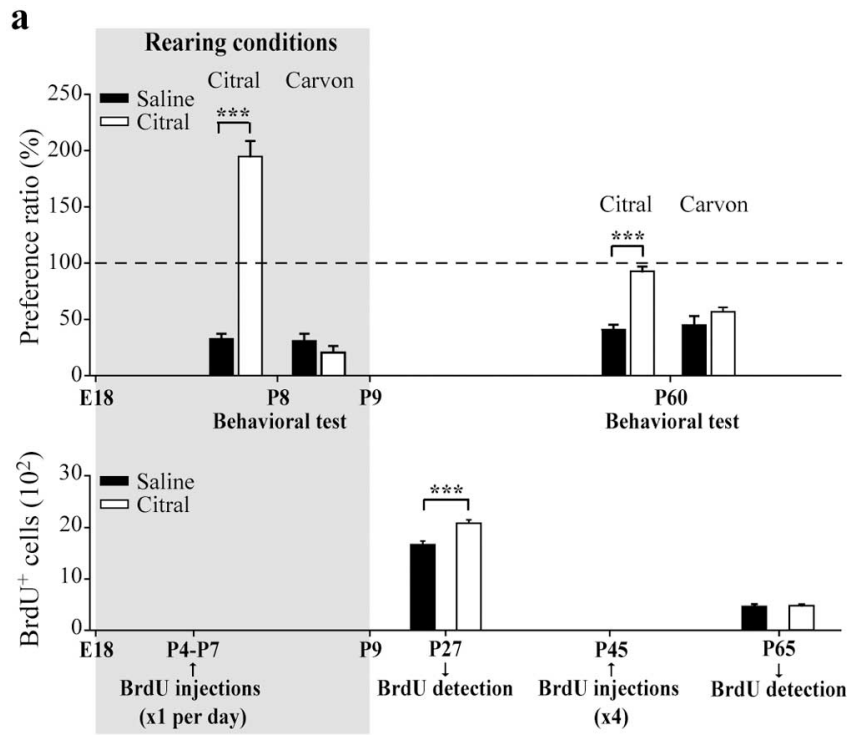

b

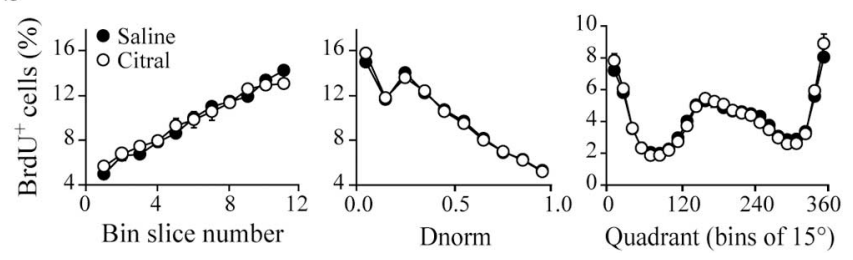

Figure 7. Early olfactory experience alters early but not late postnatal neurogenesis. $\boldsymbol{a}$, Boxed area, Pups were reared in a citral- or saline-scented environment from E18 to P9. Top graph, P8 and P60 mice were subjected to a preference test. Histograms indicate the ratio of time spent by saline- and citral-reared animals (black and white bars, respectively) investigating citral or carvon shavings versus clean shavings ( $n=9-33$ animals per group). At both ages, animals show a significant preference to citral compared with saline. The specificity of this preference is shown by the absence of preference to carvon shavings at any age. Bottom graph, Quantification of BrdU + cells following early olfactory experience. BrdU was administered either from P4 to P7 (one injection per day) or at P45 (four injections), and animals were killed for immunohistochemistry $20 \mathrm{~d}$ later. The mean number of newborn GCs was significantly higher in citral-reared animals compared with control mice when measured at P27 but not at P65. $\boldsymbol{b}$, Spatial distribution of $\mathrm{BrdU}^{+}$cells counted in the granule cell layer along the rostrocaudal axis (left panel), the internal-external axis (middle panel), and the ventrodorsal axis (right panel) of the $0 B$ taken from saline- or citral-reared mice. Animals were injected with BrdU from P4 to P7 ( $n=5-7$ animals per group). ${ }^{* *} p<0.001$ with a Student's $t$ test.

possible to change the number of early newborn neurons without affecting the number of cells generated in adulthood and this through early olfactory experience.

Because newborn cells reach the postnatal OB from its caudal region, an unexpected result to emerge from our investigations is the uniform distribution of these cells from the rostral to the caudal part of the OB. Within the bulb, active processes might therefore be taking place to actively distribute newborn cells throughout the rostrocaudal axis. However, once new GCs have reached their targeted layer, they disperse unevenly. We found that neonatal cells, generated during the first postnatal week, were preferentially targeted to the superficial part of the GCL, whereas late-born cells were restricted to the deeper part. This gradient might result from differences in the recruitment of newborn cells to the GCL rather than from a regionalization of cell death, because apoptosis was uniform across the layer.

Previous studies have reported distinct types of newborn GCs according to their location (Luskin, 1993; Baker et al., 2001; Carleton et al., 2003); however, to our knowledge, this is the first study mentioning a developmental regulation of the laminar or- ganization of the GCL. Different molecules may regulate early postnatal (or embryonic) and adult stem cell niches. Supporting this assumption are recent results showing that mice deficient in Bmi or sonic hedgehog all exhibit significant deficits in postnatal stem cell niches but not in embryos (Molofsky et al., 2003; Palma et al., 2005). Therefore, a combination of common and distinct pathways may regulate the production of neuronal precursors in the neonate and in the adult.

The selective targeting of newborn interneurons might have important functional consequences for the OB circuit. We demonstrated previously that the electrophysiological properties of superficial newborn GCs substantially differ from those located deeper (Carleton et al., 2003), and we show here that newborn GCs produced during early postnatal life possess a much higher survival rate than their adult-generated counterparts (Fig. 6b). Our observations indicating that the formation of long-term olfactory memory during early postnatal life is accompanied by an increased level of early, but not late, postnatal neurogenesis support this view. Together, these results demonstrate, for the first time, that early postnatal neurogenesis differs significantly from adult neurogenesis and highlight the possibility that newborn GCs might have distinct functional properties across different ages.

\section{Sites of production and migration rates of newborn neurons as a function of age}

Bulbar GABAergic interneurons are mainly produced postnatally (Mirich et al., 2002). Approximately 25\% of these neurons are generated during embryogenesis, $55 \%$ and $18 \%$ during the first and second postnatal week, respectively, and the remaining $2 \%$ are born during the third postnatal week to adulthood (Hinds, 1968; Bayer, 1983). Here, we found notable differences in the degree of local cell production within the $\mathrm{OB}$ across ages. Before P7, the local production of newborn cells in the bulb contributes highly to the overall amount of new GCs generated at this age, although it might also provide the $\mathrm{OB}$ with glial cells. This local proliferation decreases thereafter, becoming insignificant in adults, and leaving the SVZ/RMS production site as the unique source providing neuroblasts during later stages. Previous studies have reported a higher rate of postnatal proliferation in the SVZ compared with the local production within the OB (Hinds, 1968; Altman, 1969; Kaplan et al., 1985; Frazier-Cierpial and Brunjes, 1989), but none have determined the relative contribution between the two sites. Our finding indicates that most postnatally generated neurons are produced at locations caudal to the bulb and subsequently migrate forward.

Surprisingly, we found that newborn cells reach the OB faster in adults compared with the neonates, despite the constant growth and reorganization of the SVZ-RMS-OB pathway in the developing forebrain. Such age-dependent differences might be explained by variations in the migration speed of neuroblasts at different ages. Neuroblasts generated in the SVZ migrate to the OB tangentially in chains enwrapped by the glial tube (a tunnellike structure formed by astrocytes). Although tangential migration begins at birth, the glial tube does not become obvious until the third postnatal week (Peretto et al., 1997, 1999). Before this time window, the glial processes of astrocytes form a relatively homogeneous network. As a consequence, cells migrating in the RMS of neonates encounter more physical constraints than cells migrating in chains in adults. Furthermore, it has been demonstrated that astrocytes forming the glial tubes release molecules to promote and accelerate the migration of neuroblasts (Mason et al., 2001). This may also explain the faster migration of newborn 
neurons in the adult brain. Furthermore, the signaling mechanisms used by migrating neuroblasts in the developing and adult brain could also differ substantially. Indeed, it has been demonstrated that the developmentally regulated expression of some integrins might affect the migration behavior of neuroblasts in an age-dependent manner (Murase and Horwitz, 2002). In addition, the level of signaling molecules known to reduce migration speed, such as GABA spillover (Bolteus and Bordey, 2004), might be higher in the developing brain than in the adult because of a late onset of uptake mechanisms. Altogether, these previous studies indicate that the microenvironment surrounding neuroblasts located in the SVZ/RMS migratory pathway, as well as the expression of some membrane-bound signaling molecules and intracellular cascade mechanisms, changes drastically during early postnatal life. These changes may well explain the different migration time required by neuroblasts to reach the $\mathrm{OB}$ in neonates and young adults.

One of the most intriguing differences between newborn cells generated in the neonatal period and in adulthood is their distinct targeting in the external-internal axis of the GCL. Such targeting differences may produce important differences in the functional properties of newborn interneurons. Indeed, GABAergic interneurons positioned at different depths within the GCL might have distinct neuronal targets, because the length of their proximal dendritic tree remains constant (Orona et al., 1983). In line with this, superficial and deep GCs differ with regards to the distribution of their dendritic tree (Orona et al., 1983) and their synaptic inputs (Orona et al., 1984; Kishi et al., 1984; Carleton et al., 2003). For instance, our previous electrophysiological recordings have shown that newborn neurons, the cell body of which resides close to the mitral cell layer, were clearly distinguished from deeper ones with a cell body closer to the core of the OB (Carleton et al., 2003). The decay time of inhibitory synaptic events was consistently slower in superficial newborn GCs compared with deeper ones. This discrepancy in synaptic connectivity might result from differences in the composition of the postsynaptic receptors and/or modalities in neurotransmitter release from the presynaptic site. Not only do deeply situated GCs arborize their dendrites in the deep portion of the EPL to contact the secondary dendrites of mitral cells, but they might also contact other GCs. In contrast, superficial GCs preferentially impinge onto the secondary dendrites of tufted cells within the superficial EPL (for review, see Mori, 1987).

It is therefore possible that deep GCs, through their contacts with both mitral cells and other GCs, function to control the excitatory-inhibitory balance of the bulbar circuit. A similar idea has also been proposed for the whole population of deep shortaxon cells (Schneider and Macrides, 1978), which are thought to provide pathways for disinhibition of mitral and tufted cells via inhibitory axosomatic and axodendritic synapses on GABAergic GCs (Macrides et al., 1985). Because deep GCs establish dendrodendritic synapses predominantly with the lateral dendrites of mitral cells, they might also directly modulate their oscillatory activity. In fact, we have shown previously that dendrodendritic synapses between mitral cells and GCs play a key role in synchronizing mitral cells and in determining the oscillatory power of the OB network (Lagier et al., 2004; for review, see Saghatelyan et al., 2003). Therefore, the population of deep GCs might constitute an important component of the circuitry that participates in inducing odor-induced oscillations in mitral cell activity. In contrast, superficial GCs might provide the means for lateral inhibition of tufted cells. Furthermore, because tufted and mitral cells show distinct patterns of axonal projections to the olfactory cortex
(Skeen and Hall, 1977; Scott et al., 1980; Schoenfeld and Macrides, 1984), it is possible that GC subtypes that contact either mitral or tufted cells handle different aspects of odorant information. Given that tufted cells are adapted to detect low concentrations of odorants (Nagayama et al., 2004), we propose that early born GCs might preferentially regulate this function, whereas late born interneurons might rather control odorant identity by participating in the odor-specific temporal shaping of mitral cell spike responses.

Our results not only reveal differences in the distribution of newborn cells produced at distinct ages of life but also describe dramatic changes in their survival rate. It is thus conceivable that the population of GCs produced during the early postnatal period, and impinging onto tufted cells, remains constant throughout the entire life span of the animal. In contrast, GCs produced at later stages, which in their deeper position within the GCL will contact both mitral cells and other GCs, are renewed throughout adulthood.

\section{Survival and death of newborn and existing GCs}

We have demonstrated that the survival rate of newborn neurons differs substantially across ages. As reported by others (Petreanu and Alvarez-Buylla, 2002; Winner et al., 2002), we found that a number of adult newborn cells were eliminated within a few weeks after their arrival in the OB. This result contrasts with observations made at early stages, when almost the entire population of early generated postnatal GCs survived until adulthood. This is rather surprising, because during prenatal and early postnatal brain development, neurogenesis is usually counterbalanced by a massive level of apoptotis (Benn and Woolf, 2004). Indeed, despite the drastic differences in the survival rate of newborn GCs across ages, the overall number of TUNEL ${ }^{+}$cells in the $\mathrm{OB}$ of young and adult animals was very similar in our experiments.

Recent studies performed in the adult OB suggested that not only old neurons but also young neurons undergo apoptosis (Winner et al., 2002). It is noteworthy that TUNEL ${ }^{+}$cells in the GCL have been shown to be neurons and not astrocytes in both the developing (Najbauer and Leon, 1995; Fiske and Brunjes, 2001) and the mature (Biebl et al., 2000) OB. The differences in the survival rates of newborn neurons produced during distinct periods of life suggest distinct functional implications. During early postnatal periods, new neurons continue to be added to the developing OB, whereas in adulthood, they can be used to replace pre-existing dying neurons.

\section{Functional implications for the two postnatal neurogenic waves}

It should be noted that the population of GCs demonstrating a high survival rate is produced during the critical period of animal development when olfaction plays a vital role in the growth and survival of pups. We propose that early born GCs showing lowcell turnover represent the building blocks for $\mathrm{OB}$ functioning, linked with vital and long-lasting olfactory behaviors, whereas GCs generated during adulthood and endowed with short-term survival might rather participate in a fine-tuning of olfactory abilities (Gheusi et al., 2000; Rochefort et al., 2002; Enwere et al., 2004; for review, see Lledo and Gheusi, 2003). Our analysis of citral-reared animals indicates that, following early olfactory experience, the formation of long-term memories is accompanied by an increase in the number of early generated GCs. Supporting this notion is a previous study showing increased number of bulbar neurons subsequent to early olfactory learning (Woo and 
Leon, 1991). Although these data remain correlative, they might be supported by the different survival rates seen between neonates and adults.

Early experience plays an essential role in shaping the architecture of the brain, such that in adulthood, the brain is capable of optimally processing the range of stimuli that an animal encounters in its environment. Our results show that early and late neurogenesis might fulfill these functions through distinct modalities.

\section{References}

Altman J (1969) Autoradiographic and histological studies of postnatal neurogenesis. IV. Cell proliferation and migration in the anterior forebrain, with special reference to persisting neurogenesis in the olfactory bulb. J Comp Neurol 137:433-457.

Altman J, Das GD (1966) Autoradiographic and histological studies of postnatal neurogenesis. I. A longitudinal investigation of the kinetics, migration and transformation of cells incorporating tritiated thymidine in neonate rats, with special reference to postnatal neurogenesis in some brain regions. J Comp Neurol 126:337-389.

Baker H, Liu N, Chun HS, Saino S, Berlin R, Volpe B, Son JH (2001) Phenotypic differentiation during migration of dopaminergic progenitor cells to the olfactory bulb. J Neurosci 21:8505-8513.

Bayer SA (1983) ${ }^{3} \mathrm{H}$-Thymidine-radiographic studies of neurogenesis in the rat olfactory bulb. Exp Brain Res 50:329-340.

Belluzzi O, Benedusi M, Ackman J, LoTurco JJ (2003) Electrophysiological differentiation of new neurons in the olfactory bulb. J Neurosci 23:10411-10418.

Benn SC, Woolf CJ (2004) Adult neuron survival strategies-slamming on the brakes. Nat Rev Neurosci 5:686-700.

Betarbet R, Zigova T, Bakay RA, Luskin MB (1996) Dopaminergic and GABAergic interneurons of the olfactory bulb are derived from the neonatal subventricular zone. Int J Dev Neurosci 14:921-930.

Biebl M, Cooper CM, Winkler J, Kuhn HG (2000) Analysis of neurogenesis and programmed cell death reveals a self-renewing capacity in the adult rat brain. Neurosci Lett 291:17-20.

Bolteus AJ, Bordey A (2004) GABA release and uptake regulate neuronal precursor migration in the postnatal subventricular zone. J Neurosci 24:7623-7631.

Carleton A, Petreanu LT, Lansford R, Alvarez-Buylla A, Lledo PM (2003) Becoming a new neuron in the adult olfactory bulb. Nat Neurosci 6:507-518.

Doetsch F, Petreanu L, Caille I, Garcia-Verdugo JM, Alvarez-Buylla A (2002) EGF converts transit-amplifying neurogenic precursors in the adult brain into multipotent stem cells. Neuron 36:1021-1034.

Enwere E, Shingo T, Gregg C, Fujikawa H, Ohta S, Weiss S (2004) Aging results in reduced epidermal growth factor receptor signaling, diminished olfactory neurogenesis, and deficits in fine olfactory discrimination. J Neurosci 24:8354-8365.

Fiske BK, Brunjes PC (2001) Cell death in the developing and sensorydeprived rat olfactory bulb. J Comp Neurol 431:311-319.

Frazier-Cierpial L, Brunjes PC (1989) Early postnatal cellular proliferation and survival in the olfactory bulb and rostral migratory stream of normal and unilaterally odor-deprived rats. J Comp Neurol 289:481-492.

Gheusi G, Cremer H, McLean H, Chazal G, Vincent JD, Lledo PM (2000) Importance of newly generated neurons in the adult olfactory bulb for odor discrimination. Proc Natl Acad Sci USA 97:1823-1828.

Gritti A, Bonfanti L, Doetsch F, Caille I, Alvarez-Buylla A, Lim DA, Galli R, Verdugo JM, Herrera DG, Vescovi AL (2002) Multipotent neural stem cells reside into the rostral extension and olfactory bulb of adult rodents. J Neurosci 22:437-445.

Hinds JW (1968) Autoradiographic study of histogenesis in the mouse olfactory bulb. I. Time of origin of neurons and neuroglia. J Comp Neurol 134:287-304

Kaplan MS, Hinds JW (1977) Neurogenesis in the adult rat: electron microscopic analysis of light radioautographs. Science 197:1092-1094.

Kaplan MS, McNelly NA, Hinds JW (1985) Population dynamics of adultformed granule neurons of the rat olfactory bulb. J Comp Neurol 239:117-125.

Katz LC, Shatz CJ (1996) Synaptic activity and the construction of cortical circuits. Science 274:1133-1138.
Kishi K, Mori K, Ojima H (1984) Distribution of local axon collaterals of mitral, displaced mitral, and tufted cells in the rabbit olfactory bulb. J Comp Neurol 225:511-526.

Lagier S, Carleton A, Lledo PM (2004) Interplay between local GABAergic interneurons and relay neurons generates gamma oscillations in the rat olfactory bulb. J Neurosci 24:4382-4392.

Lledo PM, Gheusi G (2003) Olfactory processing in a changing brain. NeuroReport 14:1655-1663.

Lois C, Alvarez-Buylla A (1994) Long-distance neuronal migration in the adult mammalian brain. Science 264:1145-1148.

Luskin MB (1993) Restricted proliferation and migration of postnatally generated neurons derived from the forebrain subventricular zone. Neuron 11:173-189.

Macrides F, Schoenfeld TA, Marchand JE, Clancy AN (1985) Evidence for morphologically, neurochemically and functionally heterogeneous classes of mitral and tufted cells in the olfactory bulb. Chem Senses 10:175-202.

Mason HA, Ito S, Corfas G (2001) Extracellular signals that regulate the tangential migration of olfactory bulb neuronal precursors: inducers, inhibitors, and repellents. J Neurosci 21:7654-7663.

Mechawar N, Saghatelyan A, Grailhe R, Scoriels L, Gheusi G, Gabellec MM, Lledo PM, Changeux JP (2004) Nicotinic receptors regulate the survival of newborn neurons in the adult olfactory bulb. Proc Natl Acad Sci USA 101:9822-9826.

Mirich JM, Williams NC, Berlau DJ, Brunjes PC (2002) Comparative study of aging in the mouse olfactory bulb. J Comp Neurol 454:361-372.

Molofsky AV, Pardal R, Iwashita T, Park IK, Clarke MF, Morrison SJ (2003) Bmi-1 dependence distinguishes neural stem cell self-renewal from progenitor proliferation. Nature 425:962-967.

Mori K (1987) Membrane and synaptic properties of identified neurons in the olfactory bulb. Prog Neurobiol 29:275-320.

Murase S, Horwitz AF (2002) Deleted in colorectal carcinoma and differentially expressed integrins mediate the directional migration of neural precursors in the rostral migratory stream. J Neurosci 22:3568-3579.

Nagayama S, Takahashi YK, Yoshihara Y, Mori K (2004) Mitral and tufted cells differ in the decoding manner of odor maps in the rat olfactory bulb. J Neurophysiol 91:2532-2540.

Najbauer J, Leon M (1995) Olfactory experience modulates apoptosis in the developing olfactory bulb. Brain Res 674:245-251.

Najbauer J, Yan XX, Leon M (2002) Internucleosomal DNA fragmentation during deprived and non-deprived olfactory development. Brain Res 926:118-125.

Olivo-Marin JC (2002) Extraction of spots in biological images using multiscale products. Pattern Recog 35:1989-1996.

Orona E, Scott JW, Rainer EC (1983) Different granule cell populations innervate superficial and deep regions of the external plexiform layer in rat olfactory bulb. J Comp Neurol 217:227-237.

Orona E, Rainer EC, Scott JW (1984) Dendritic and axonal organization of mitral and tufted cells in the rat olfactory bulb. J Comp Neurol 226:346-356.

Palma V, Lim DA, Dahmane N, Sanchez P, Brionne TC, Herzberg CD, Gitton Y, Carleton A, Alvarez-Buylla A, Ruiz i Altaba A (2005) Sonic hedgehog controls stem cell behavior in the postnatal and adult brain. Development 132:335-344.

Pencea V, Luskin MB (2003) Prenatal development of the rodent rostral migratory stream. J Comp Neurol 463:402-418.

Peretto P, Merighi A, Fasolo A, Bonfanti L (1997) Glial tubes in the rostral migratory stream of the adult rat. Brain Res Bull 42:9-21.

Peretto P, Merighi A, Fasolo A, Bonfanti L (1999) The subependymal layer in rodents: a site of structural plasticity and cell migration in the adult mammalian brain. Brain Res Bull 49:221-243.

Petreanu L, Alvarez-Buylla A (2002) Maturation and death of adult-born olfactory bulb granule neurons: role of olfaction. J Neurosci 22:6106-6113.

Rochefort C, Gheusi G, Vincent JD, Lledo PM (2002) Enriched odor exposure increases the number of newborn neurons in the adult olfactory bulb and improves odor memory. J Neurosci 22:2679-2689.

Rodriguez Echandia EL, Foscolo M, Broitman ST (1982) Preferential nesting in lemon-scented environment in rats reared on lemon-scented bedding from birth to weaning. Physiol Behav 29:47-49. 
Rosselli-Austin L, Altman J (1979) The postnatal development of the main olfactory bulb of the rat. J Dev Physiol 1:295-313.

Saghatelyan A, Carleton A, Lagier S, de Chevigny A, Lledo PM (2003) Local neurons play key roles in the mammalian olfactory bulb. J Physiol (Paris) 97:517-528.

Schneider SP, Macrides F (1978) Laminar distributions of interneurons in the main olfactory bulb of the adult hamster. Brain Res Bull 3:73-82.

Schoenfeld TA, Macrides F (1984) Topographic organization of connections between the main olfactory bulb and pars externa of the anterior olfactory nucleus in the hamster. J Comp Neurol 227:121-135.

Scott JW, McBride RL, Schneider SP (1980) The organization of projections from the olfactory bulb to the piriform cortex and olfactory tubercle in the rat. J Comp Neurol 194:519-534.
Shah A, Oxley G, Lovic V, Fleming AS (2002) Effects of preweaning exposure to novel maternal odors on maternal responsiveness and selectivity in adulthood. Dev Psychobiol 41:187-196.

Skeen LC, Hall WC (1977) Efferent projections of the main and the accessory olfactory bulb in the tree shrew (Tupaia glis). J Comp Neurol 172:1-35.

Voyvodic JT (1996) Cell death in cortical development: how much? Why? So what? Neuron 16:693-696.

Winner B, Cooper-Kuhn CM, Aigner R, Winkler J, Kuhn HG (2002) Longterm survival and cell death of newly generated neurons in the adult rat olfactory bulb. Eur J Neurosci 16:1681-1689.

Woo CC, Leon M (1991) Increase in a focal population of juxtaglomerular cells in the olfactory bulb associated with early learning. J Comp Neurol 305:49-56. 